\title{
Endogenous Adult-Derived Telomerase-Positive Stem Cells
}

\author{
Henry E Young* \\ Dragonfly Foundation for Research and Development, USA
}

\begin{abstract}
ABBREVIATIONS: AD: Alzheimer's Disease; ALS: Amyotrophic Lateral Sclerosis; B: Blindness; CIDP: Chronic Inflammatory Demyelinating Polyneuropathy; D: Dementia; MD: Macular Degeneration; MS: Multiple Sclerosis; N: Neuropathies; PD: Parkinson Disease; SC: Sciatica; ST: Stroke; TBI: Traumatic Brain Injury; TSCI: Traumatic Spinal Cord Injury
\end{abstract}

\section{INTRODUCTION}

Discovered in 1975 Located in the connective tissues throughout the body, 10\% of all stem cells in the body Found in 16 different species: terrestrial salamander, Komodo dragon, chicken, waddle crane, mouse, rat, rabbit, cat, dog, sheep, goat, pig, cow, speckled bear, horse, and human They are the natural healing cells of the body, they do NOT decrease in number with increasing age of the individual Cloned from single cells by repetitive serial dilution clonogenic analysis and then extensively characterized Consist of the following (Figure $1 \& 2$ )

- Totipotent stem cells-form any cell type in the body, including spermatogonia and oogonia

- Pluripotent stem cells-form any cell type in the body, except spermatogonia and oogonia Ectodermal stem cells-will only form cells of the ectodermal embryonic lineage

- Mesodermal stem cells-will only form cells of the mesodermal embryonic lineage

- Endodermal stem cells- will only form cells of the endodermal embryonic lineage Utilized for treatments

Will NOT form teratomas (cancerous cells) in situ or ex vivo. Normally quiescent, unless activated. Inside the body, activation requires about 2 weeks Outside the body, activation takes 2 hours. Can be expanded to significant numbers inside the body (in situ) using combinatorial nutraceuticals and outside the body (ex vivo) in culture using proprietary culture media: Essentially unlimited proliferation potential. Transplanted either as autologous (self) and/or allogeneic (donor)Donor needs to be matched for gender, ABO blood group (or O-negative), infectious disease-free and deleterious genetic mutation-free.

Telomerase-positive stem cells used to treat following conditions:

Neurodegenerative (TSCs - intranasal, PSCs \& MesoSCs IV): Alzheimer's Disease (AD), Amyotrophic

Lateral Sclerosis (ALS), Blindness (B), Chronic Inflammatory Demyelinating Polyneuropathy (CIDP), Dementia (D), Macular Degeneration (MD), Multiple Sclerosis (MS), Neuropathies (N), Parkinson Disease, (PD) Sciatica (SC), Stroke (ST), Traumatic Brain Injury (TBI), Traumatic Spinal Cord Injury (TSCI).

Cardiovascular (TSCs - slow IV; PSCs \& MesoSCs regular IV): Myocardial Infarction (MI)

Pulmonary (TSCs \& PSCs - nebulized; MesoSCs - regular IV): Chronic Obstructive Pulmonary

Disease (COPD), Interstitial Pulmonary Fibrosis (IPF) - survived up to eight years after initiation of treatment
Quick Response Code:

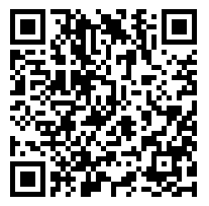

Address for correspondence: Henry E Young, Dragonfly Foundation for Research and Development, USA

Received: January 28, 2020

Published: February 11, 2020

How to cite this article: Henry E Young. Endogenous Adult-Derived Telomerase-Positive Stem Cells. 2020 - 2(1) OAJBS.ID.000139. DOI: 10.38125/OAJBS.000139 
Orthopedic (TSCs, PSCs \& MesoSCs - direct inject; TSCs, PSCs \& MesoSCs - regular IV): Osteoarthritis (OA), Muscle Atrophy (MA), Wound Healing (WH),

Autoimmune (TSCs - intranasal; TSCs - slow IV; TSCs \& PSCs - nebulized; TSCs, PSCs \& MesoSCs - regular IV): Celiac Disease (CD), Systemic Lupus Erythematosus (SLE), stage-IV - still alive nine years and counting

Anti-Aging (TSCs \& PSCs - direct inject; TSCs, PSCs \& MesoSCs - regular IV): Wrinkles Cosmetic [4] (TSCs, PSCs \& MesoSCs - regular IV): Hair color back to original color of individual providing stem cells, either self or donor.
Combinatorial Nutraceuticals - increase endogenous adult telomerase-positive stem cells in situ. Positive results seen in protocol-compliant individuals for at least 1-2 months posttransplant, some as long as eight years post-transplant. We use three types of endogenous adult-derived telomerase-positive stem cells for our therapies: telomerase-positive mesodermal stem cells (MesoSCs), pluripotent stem cells (PSCs), and totipotent stem cells (TSCs). The MesoSCs have the capability to form at least 37 separate and distinct cell types within the mesodermal embryonic germ line lineage. The PSCs have the capability to form all cells within the individual EXCEPT the germ cells (sperm and ova) and notochord. And the TSCs have the capability of forming ALL cell types within the individual, including the germ cells and notochord (Figure 1).

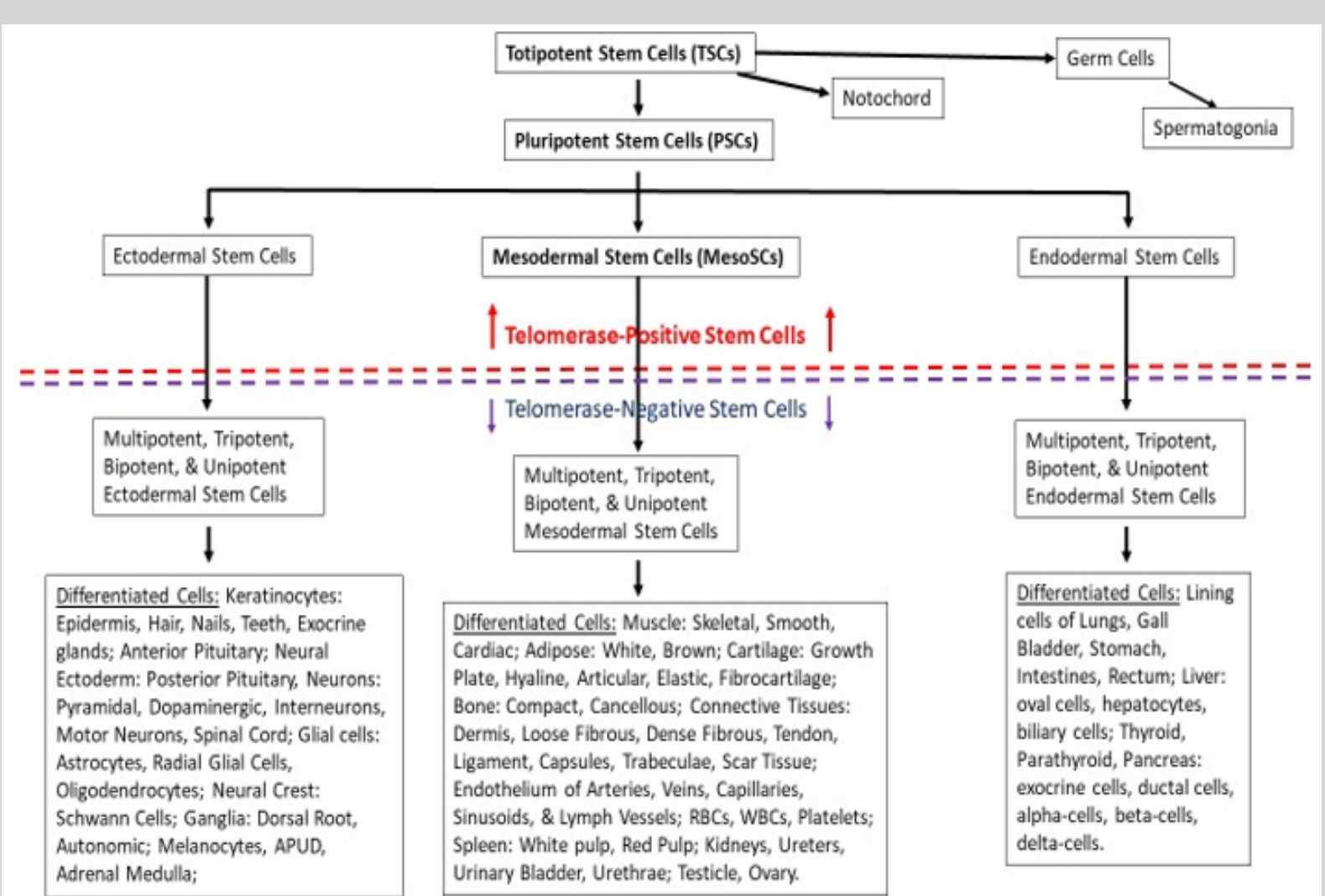

Figure 1: Diagram of telomerase-positive stem cells (with an essentially unlimited proliferation potential) [above red dashed line] and telomerase-negative stem cells (which conform to Hayflick's limit of 50-70 population doublings before senescence and death) [below blue dashed line] within the body and their respective unidirectional downstream differentiation potentials.

With subsequent studies we discovered that the telomerasepositive totipotent stem cells are equivalent in differentiation potential to the blastomeres of the morula during embryonic development of an individual (Figure 2), forming all tissues of the individual, including the germ cells (sperm and ova) and the notochord. The telomerase-positive pluripotent stem cells are equivalent in differentiation potential to the embryonic epiblast, forming all tissues of the individual except the germ cells and notochord; the telomerase-positive mesodermal stem cells are equivalent in differentiation potential to the embryonic mesoderm germ layer lineage, forming all tissues within the mesodermal lineage, but will NOT cross over to form tissues of the ectodermal or endodermal lineages; the telomerase-positive ectodermal stem cells are equivalent in differentiation potential to the embryonic ectoderm germ layer lineage, forming all tissues within the ectodermal lineage, but will NOT cross over to form tissues of the mesodermal or endodermal lineages; and the telomerase-positive endodermal stem cells are equivalent in differentiation potential to the embryonic endoderm germ layer lineage, forming all tissues within the endodermal lineage, but will NOT cross over to form tissues of the ectodermal or mesodermal lineages. Based on Figures $1 \& 2$, we designed the in situ proliferation, in situ mobilization, harvest by venipuncture, separation using minimally manipulative procedures (zeta potential, density, gravity, differential centrifugation with serum gradients and aqueous gradients), activation, and treatment techniques designed specifically for telomerase-positive totipotent stem cells, pluripotent stem cells, and mesodermal stem cells. 


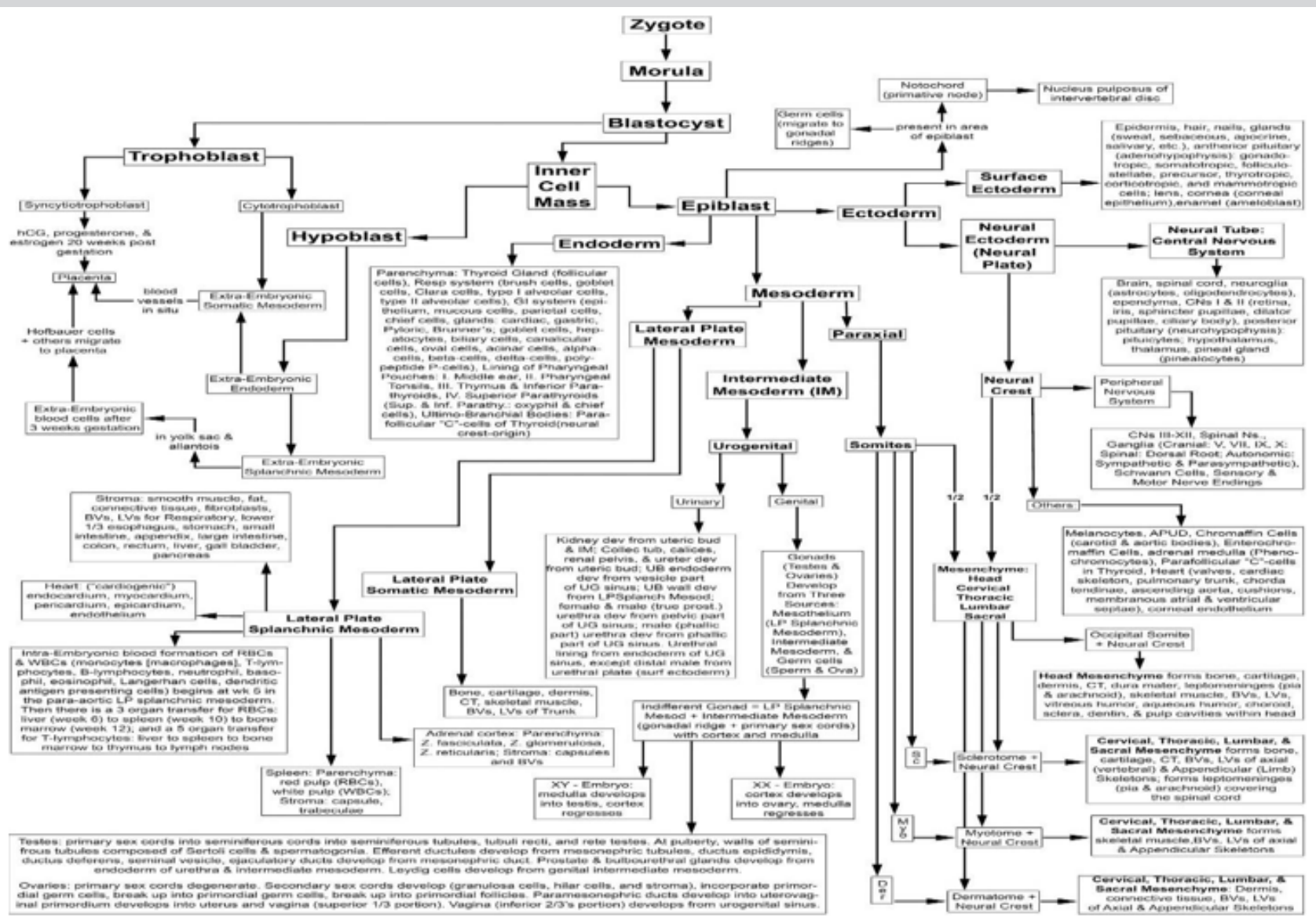

Figure 2: Lineage map of embryonic development. Reprinted with permission from Young HE, Black AC Jr. Adult Stem Cells. Anatomical Record 276A:75-102, 2004.

\section{ENDOGENOUS TELOMERASE-POSITIVE ADULT STEM CELLS}

1. Age range for presence of endogenous telomerase-positive stem cells is newborn through geriatric-aged individuals.

2. Telomerase-positive adult stem cells do NOT decrease in number with increasing age of the individual.

3. Telomerase-positive adult stem cells are present in both males and females.

4. Telomerase-positive adult stem cells are located in all connective tissues throughout the body.

5. Telomerase-positive adult stem cells, e.g., TSCs, PSCs, MesoSCs, EctoSCs, and EndoSCs, comprise 10\% of all (telomerasepositive and telomerase-negative) stem cells throughout the individual.

6. TSCs comprise $0.1 \%$ of all (telomerase-positive and telomerase-negative) stem cells within an individual.

7. PSCs comprise $0.9 \%$ of all (telomerase-positive and telomerase-negative) stem cells within an individual.

8. MesoSCs comprise $3.0 \%$ of all (telomerase-positive and telomerase-negative) stem cells within an individual.

9. [EctoSCs comprise $3.0 \%$ of all (telomerase-positive and telomerase-negative) stem cells within an individual.]
10. [EndoSCs comprise $3.0 \%$ of all (telomerase-positive and telomerase-negative) stem cells within an individual.]

11. Harvest and processing methods have been designed specifically for telomerase-positive adult-derived stem cells.

a. Ingestion of nutraceutical \#1 for a minimum of 30 days to induce proliferation in connective tissue- resident TSCs, PSCs, and MesoSCs.

b. Ingestion of nutraceutical \#2 18 hours before harvest to mobilize TSCs, PSCs, and MesoSCs into the blood stream.

c. Harvest whole blood containing telomerase-positive stem cells by venipuncture

d. Utilizing zeta potential, gravity, density, and differential centrifugation, including serum gradients and aqueous gradients:

e. Separate blood cells from telomerase-positive stem cells.

f. Segregate telomerase-positive stem cells into separate populations of TSCs, PSCs, and MesoSCs

g. Recombine telomerase-positive stem cells and prepare for treatments

h. Activate telomerase-positive stem cells

12. Treatment paradigms 
a. Neurodegenerative - TSCs intranasal and PSCs + MesoSCs by regular IV infusion rate.

b. Cardiovascular - TSCs by slow IV infusion and PSCs and MesoSCs by regular IV infusion rate.

c. Pulmonary - TSCs + PSCs by nebulization and MesoSCs by regular IV infusion rate.

d. Orthopedic $-1 / 2$ TSCs + PSCs + MesoSCs by direct injection into joint and $1 / 2$ TSCs + PSCs + MesoSCs by regular IV infusion.

e. Systemic - TSCs by slow IV infusion and PSCs and MesoSCs by regular IV infusion rate.

\section{ALLOGENEIC TELOMERASE-POSITIVE ADULT STEM CELLS}

1. Are comprised of allogeneic (non-self) telomerase-positive totipotent stem cells and pluripotent stem cells, only.

2. Telomerase-positive stem cell-specific characteristics are described above.

3. The donor of allogeneic stem cells needs to be tested for the infectious diseases.

4. The donor of allogeneic stem cells needs to be tested for the deleterious disease-causing genetic mutations.

5. Naïve, undifferentiated allogeneic TSCs and PSCs are transplanted into an individual they are protected from the recipient's immune system due to absence of MHC-I cell surface marker (that distinguishes self from non-self)

6. As the allogeneic TSCs and PSCs begin to differentiate and express their allogeneic MHC-I molecule on its cell surface the recipient's immune system appears to accept the cells as self and no graft versus host response occurs.

7. In contrast, MesoSCs are NOT protected from the recipient's immune system due to the presence of the allogeneic MHC-l cell surface marker, that distinguishes self from non-self, in its undifferentiated state and a graft versus host response will occur. Therefore, we do not use MesoSCs as allogeneic donor stem cells for transplant.

8. Allogeneic telomerase positive TSCs and PSCs need to be matched for gender. Gender match is significant due to the migratory ability of these particular stem cells to areas of tissue damage and the fact that each person's adrenal cortex secretes low levels of both testosterone and estrogen throughout the lifespan of the individual. We have found that "XY" telomerase-positive adult stem cells are more sensitive to testosterone than estrogen and " $\mathrm{XX}$ " telomerase-positive adult stem cells are more sensitive to estrogen than testosterone. Therefore, if male telomerase-positive stem cells transplanted into a female, when migrating into different locations than intended can express male secondary sexual characteristics. Whereas, if female telomerase-positive stem cells are transplanted into a male and migrate to different locations than intended they can express female secondary sexual characteristics.

9. Allogeneic telomerase-positive TSCs and PSCs need to be matched for $\mathrm{ABO}$ blood groups or be from an 0-negative universal donor.

10. If allogeneic TSCs are given via the intranasal route, then there is the chance for transfer of both the

\section{Donor's personality and hair color to the recipient.}

12. Side effects can occur with multiple intranasal treatments: loss of wrinkles, change of hair color to that of post-pubic stem cell provider (autologous or allogeneic).

\section{TREATMENT FOR NEURODEGENERATIVE DISEASES}

For neurological treatments, the smallest stem cells (TSCs) are in one group and the intermediate (PSCs) and larger (MesoSCs) stem cells are pooled. The (TSCs) are given by intranasal infusion. The recipient is asked to remove the mucus their nose with a saline wash prior to intranasal infusion. Because the TSCs are so small they can migrate between the nasal epithelial cells, "climb" up the olfactory nerve rootlets, "crawl" through the cribriform plate in the skull, by-passing the blood-brain barrier, "crawl" along the olfactory nerve to cisterns around and within the brain and central canal of the spinal cord, migrate to areas of damage within the brain and spinal cord and repair the damage. The PSCs and MesoSCs are given by intravenous infusion. The body has a tendency to "steal" activated stem cells and place them in areas where it thinks the need is greater than the patient would like. Therefore, we give the intravenous infusion of PSCs and MesoSCs so the body has sufficient numbers of activated stem cells for its own use.

\section{TREATMENT FOR PULMONARY DISEASES}

For pulmonary treatments, the smallest (TSCs) and the intermediate (PSCs) stem cells are pooled, and larger (MesoSCs) stem cells are processed separately. The TSCs and PSCs are given by nebulization. Because TSCs and PSCs can form all cell types within the lung, they are given in nebulized (vaporized) form and migrate to areas of damage within the lungs and trachea/bronchial tree and repair the damage. The MesoSCs are given by intravenous infusion. The body has a tendency to "steal" activated stem cells and place them in areas where it thinks the need is greater than the patient would like. Therefore, we give the intravenous- infusion of MesoSCs so the body has sufficient numbers of activated stem cells for its own use.

\section{TREATMENT FOR CARDIOVASCULAR DISEASES}

Treatment for cardiovascular disease is based on a unique architectural feature of the heart and the smallest stem cells. The heart contains endothelial-lined channels traversing the heart muscle (myocardium) between the outside layer (epicardium) and the chambers inside the heart (i.e., atria and ventricles). These channels have an opening that is less than the size of a red blood cell (7 microns). These channels transport plasma between the epicardium and the inner chambers of the heart during contraction (systole) and relaxation (diastole) of the heart muscle. The TSCs are significantly smaller (0.1-2.0 microns) than the opening of the channels and freely move back and forth in these channels during contraction and relaxation of the heart.

For cardiovascular treatments, the smallest stem cells (TSCs) are in one group and the intermediate (PSCs) and larger (MesoSCs) stem cells are pooled. The TSCs are given by a slow intravenous infusion to maximize the time the TSCs are in the vascular channels within the heart muscle (myocardium). The PSCs and MesoSCs are given by regular intravenous infusion. The body has a tendency to "steal" activated stem cells and place them in areas where it thinks the need is greater than the patient would like. Therefore, we give the IV-infusion of PSCs and MesoSCs so the body has sufficient numbers of activated stem cells for its own use. When treatment 
is completed you can go back to where you are staying near the treatment site, but you are requested to minimize activity to light levels for the next 24 hours.

\section{TREATMENT FOR AUTOIMMUNE, RENAL, AND HEPATIC DISEASES}

For autoimmune, renal, and hepatic treatments, the smallest stem cells (TSCs) are in one group and the intermediate (PSCs) and larger (MesoSCs) stem cells are pooled. The TSCs are given by a slow intravenous infusion to maximize the time the TSCs are in the organs. The PSCs and MesoSCs are given by regular intravenous infusion. The body has a tendency to "steal" activated stem cells and place them in areas where it thinks the need is greater than the patient would like. Therefore, we give the IV-infusion of PSCs and MesoSCs so the body has sufficient numbers of activated stem cells for its own use. When treatment is completed you can go back to where you are staying near the treatment site, but you are requested to minimize activity to light levels for the next 24 hours.

\section{TREATMENT FOR ORTHOPEDIC DISORDERS}

For orthopedic treatments, the smallest stem cells (TSCs), the intermediate (PSCs) and larger (MesoSCs) stem cells are pooled and then split into two equal sub-pools of cells. One sub-pool of TSCs, PSCs, and MesoSCs are given by direct injection into the joint. The other sub-pool of TSCs, PSCs, and MesoSCs are given by regular intravenous infusion. The body has a tendency to "steal" activated stem cells and place them in areas where it thinks the need is greater than the patient would like. Therefore, we give the IV-infusion of TSCs, PSCs, and MesoSCs so the body has sufficient numbers of activated stem cells for its own use.

\section{TREATMENTS TO DATE WITH TELOMERASE- POSITIVE STEM CELLS}

Alzheimer's Disease (AD) donor (allogeneic) TSCs from gender-matched, ABO-matched individuals with family histories absent of Alzheimer's disease reversed symptoms in individuals with Alzheimer's disease for a period of two months before next transplant. We were able to sustain Alzheimer's-free status through at least six treatments in protocol compliant individuals.

Amyotrophic Lateral Sclerosis (ALS) both autologous and allogeneic (gender-matched, ABO-matched) TSCs can reverse symptoms of disease for a period of one to four weeks posttransplant. Unfortunately, due to logistics could only transplant using fresh isolates once every eight weeks. Cytapheresis allows collection of greater number of stem cells, but still relegated to once every eight weeks. Currently assessing capability to expand telomerase-positive stem cells ex vivo to shorten time frame between transplants.

Blindness (B) autologous TSCs incrementally reversed total blindness to visualizing shades of gray through multiple treatments in protocol compliant individuals.

Celiac Disease allogeneic (gender-matched, ABO-matched) TSCs and PSCs completely reversed symptoms of Celiac disease for a period of eight years post-transplant in protocol compliant individual.

Chronic Inflammatory Demyelinating Polyneuropathy (CIDP) autologous TSCs reversed symptoms of disease for a period of two months or more post-transplant. We were able to sustain CIDPfree status through at least six treatments in protocol compliant individuals.
Chronic Obstructive Pulmonary Disease (COPD) autologous and allogeneic TSCs and PSCs reduced symptoms of COPD for a period of two months or more post-transplant. We were able to sustain reduced COPD symptoms for eight years in protocol compliant individuals.

Combinatorial Nutraceuticals an individual experienced a massive myocardial infarction following five CABG procedures and 15 stents, leaving him with a cardiac output of $10 \%$. Continuous treatment with combinatorial nutraceuticals only, which dispensed autologous TSCs, PSCs, and MesoSCs into his system 24/7, after four months raised his cardiac output to 35\%. After an additional four months his cardiac output was measured between $40-50 \%$.

Dementia (D) autologous TSCs reversed symptoms of dementia for a period of six months post- transplant in protocol compliant individuals.

Diabetes Type-I autologous and/or allogeneic (gender-matched, ABO-matched) TSCs and PSCs reduced insulin dependence from one to two months post-treatment in protocol compliant individuals through five years and counting.

Hair Color autologous and/or allogeneic (gender-matched, ABO-matched) restored hair color to stem cell donor's original hair color within two to six months post treatment in protocol compliant individuals.

Interstitial Pulmonary Fibrosis (IPF) autologous and allogeneic TSCs and PSCs increased lung function in individuals with IPF through eight years and counting in protocol compliant individuals.

Knee Pain a single direct injection of TSCs, PSCs, and MesoSCs into the knee joint reduced knee pain of long-standing duration from $90 \%$ to $10 \%$ for two years and counting in protocol compliant individuals.

Macular Degeneration (MD) autologous TSCs reversed symptoms of disease in protocol compliant individuals without comorbidities.

Muscle Atrophy direct injection of autologous TSCs, PSCs, and MesoSCs into atrophic muscles restored muscle activity in compliant individuals from one to four months post-injection, in protocol compliant individual.

Myocardial Infarction (MI) autologous TSCs, PSCs, and MesoSCs increased cardiac output to $45 \%$ after two treatments in an individual with a six-year sustained cardiac output of $25 \%$ following a MI.

Multiple Sclerosis (MS) autologous TSCs reversed symptoms of disease for a period of two to six months post-transplant. We were able to sustain increasing stepwise reversal of symptoms through six treatments in protocol compliant individuals.

Neuropathies (N) autologous and/or allogeneic (gendermatched, ABO-matched) TSCs reversed symptoms of disease for a period up to two months post-transplants in compliant individuals. We were able to sustain reversal of symptoms through eight years and counting in protocol compliant individuals.

Parkinson Disease (PD) autologous TSCs stabilized and/or reversed symptoms of disease for a period of 24 months posttransplant in protocol compliant individuals.

Sciatica (Sc) autologous and/or allogeneic (gender-matched, ABO-matched) TSCs reversed symptoms of Sciatica for a period of 
two to six months post-transplant in protocol compliant individuals.

Stroke (Sk) autologous TSCs incrementally reversed long standing symptoms of stroke, depending on number of treatments given in protocol compliant individuals

Systemic Lupus Erythematosus (SLE) autologous and/or allogeneic (gender-matched, ABO-matched) TSCs, PSCs, and autologous MesoSCs reversed symptoms of SLE for a period of two to 12 months post- transplant in compliant individuals, through nine years and counting in protocol compliant individual.

Traumatic Brain Injury (TBI) autologous TSCs in combination with and with/out hyperbaric oxygen incrementally reversed symptoms of TBI in protocol compliant individuals.
Traumatic Spinal Cord Injury (TSCI) autologous TSCs incrementally and permanently reversed paraplegic symptoms of TSCI in compliant individuals. One protocol compliant paraplegic individual (level T12) had bladder/bowel function restored after two autologous stem cell treatments.

Wound Healing (WH) autologous and/or allogeneic (gendermatched, ABO-matched) TSCs, PSCs, and autologous MesoSCs accelerated wound healing with minimal to no scar formation in protocol compliant individuals.

Wrinkles autologous and/or allogeneic (gender-matched, ABOmatched) TSCs, PSCs, and autologous MesoSCs restored youthful appearance of recipient for two to 12 months post treatment in protocol compliant individuals. 